\title{
MODAL PARTICIPATION FACTORS AND THEIR POTENTIAL APPLICATIONS IN AEROSPACE: A REVIEW
}

\author{
Michelle Guzman Nieto*, Mostafa S.A. ElSayed** \\ Dept. of Mechanical and Aerospace Engineering \\ Carleton University \\ Ottawa, Ontario, Canada \\ *Michelle.guzman@carleton.ca \\ **Mostafa.elsayed@carleton.ca
}

\author{
Denis Walch \\ Bombardier Aerospace \\ St-Laurent, Quebec, Canada \\ Denis.Walch@aero.bombardier.com
}

\begin{abstract}
In this paper we review different definitions of Modal Participation Factor (MPF) available in the literature and propose a simple classification and unified concept for their application with particular focus in aerospace engineering. A case study is presented to aid the reader understand under what conditions each definition of MPF applies, its physical interpretation and which definitions have the potential to act as response tracking mechanisms during design optimization.
\end{abstract}

Keywords: modal participation factor, modal contribution factors, dynamic analysis, mode tracking

\section{INTRODUCTION}

In practice, complex structures must be analyzed under a large set of dynamic operating conditions to ensure that the design will meet prescribed functional requirement.

During design optimization, a relatively small number of such operating conditions are selected as critical and are used to minimize or maximize the objective function. However, the dynamic response is strongly dependent on the physical characteristics of the structure and thus the use of a deterministic set of critical operating conditions may lead to unexpected behaviors. To monitor inexpensively the behavior of the design space during optimization, a response tracking mechanism based on MPFs could lead to large computational savings and improve the optimization process by considering the variations of the response of the design space as the design evolves.

MPFs have been used in the past as response tracking mechanisms in several engineering disciplines. For instance, Keong et al. [1] tracked a targeted mode during the design optimization of a head suspension assembly by defining a participation factor that resembled the most to the mode of interest. In the field of civil engineering, MPFs have been widely used to approximate the dynamic response of buildings without performing any dynamic analysis [2-13]; this procedure was later on applied in aerospace for the case of a cantilever wing model [14].

The concept of MPF, however, seems to be ambiguous. A simple search of the words "modal participation factor" in the open literature quickly reveals that several variants of this concept exist. For instance, in electrical engineering [15-18] the term MPF refers to the product between the left and the right eigenvectors; other researchers [19-22] used simple definitions for MPF such as the same term to refer to the components of the eigenvector matrix. On the other hand, [23-28] defined different convenient mathematical forms as modal participation factor suitable to their specific applications.

This significant variation in the definitions of MPFs is also conveys into a wide range of applications. For instance, Wallrapp \&Wiedemann [29] computed a quasi-static modal solution and a corresponding participation factor to assess the importance of each mode retained in the solution; Chung-Band and Young Bahng [30] utilized a participation factor based on modal strain energy to identify dominant modes; Van Lagenhove \&Brughmans [31] reviewed three kinds of MPFs as methods to identify the best location for sensors to correlate a Finite Element Model (FEM) with experimental results. Similarly, other researchers [32,33] used different definitions of MPFs to correlate experimental with numerical results.

In this paper, we propose a unified concept and a simple classification of the different mathematical definitions of modal participation factors found in the literature. A case study is presented to aid the reader to understand under what conditions each definition applies, its physical interpretation and which definitions have the potential to act as response tracking mechanisms during design optimization.

\section{MODAL CONTRIBUTION FACTORS}

The complex behavior of a dynamic structure can be decomposed into a set of simple harmonic motions $\mathrm{q}_{\mathrm{i}}(\mathrm{t})$, also known as modal responses, linearly coupled with amplitude ratios. This transformation is achieved by finding the so-called natural frequencies, $\omega_{\mathrm{r}}$ and mode shapes, $\Phi_{\mathrm{r}}$ that satisfy the characteristic equation (i.e., the undamped free vibration problem) and applying the following variable change:

$$
\mathrm{u}(\mathrm{t})=\Phi \mathrm{q}(\mathrm{t})
$$

Where $\mathrm{u}(\mathrm{t})$ is the nodal vector of physical displacements, and $\Phi$ is the matrix of eigenvectors. Expanding (1) in a summation form we obtain: 


$$
\mathrm{u}_{\mathrm{n}}(\mathrm{t})=\phi_{\mathrm{n}, 1} \mathrm{q}_{1}(\mathrm{t})+\ldots+\phi_{\mathrm{n}, \mathrm{i}} \mathrm{q}_{\mathrm{i}}(\mathrm{t})
$$

From (2), the modal contribution can be defined as the quantity of movement that each mode contributes to the total nodal response [34-37]. In other words, it is the product between the modal response $\mathrm{q}_{\mathrm{i}}(\mathrm{t})$ and the amplitude ratio $\phi_{\mathrm{n}, \mathrm{i}}$, denoted here with the Greek letter gamma $(\gamma)$.

$$
\gamma_{\mathrm{n}, \mathrm{i}}(\mathrm{t})=\phi_{\mathrm{n}, \mathrm{i}} \mathrm{q}_{\mathrm{i}}(\mathrm{t})
$$

All definitions of modal participation factors aim to rank the relative importance of $\gamma_{\mathrm{n}, \mathrm{i}}$, nevertheless, these definitions can be subdivided in two classes. The first class (Class I) is derived from the equations of motion and it's commonly known as static modal participation factors (SMPF). SMPFs have the potential to be used as a reanalysis tool since they predict the magnitude of $\mathrm{q}_{\mathrm{i}}$ [42-51]. The second class (Class II) is intended solely to assess the relative importance of a mode within the solution of a system response.

We should make emphasis on the term "relative importance of a mode", since the interpretation of the results obtained from the second class of participation factors is dependent on its mathematical definition, as discussed below in section IV.

\section{CLASS I}

The amplitude of a modal response can be expressed as the product of a constant called modal participation factor $\Gamma_{\mathrm{i}}$ and a time dependent term $\mathrm{D}_{\mathrm{i}}(\mathrm{t})[7,14,36,37]$.

$$
\mathrm{q}_{\mathrm{i}}(\mathrm{t})=\Gamma_{\mathrm{i}} \mathrm{D}_{\mathrm{i}}(\mathrm{t})
$$

Though in general the time history of the response may be of interest, it is the value of the maximum displacement that will be used to designing or optimizing a structure. The maximum value of the response $\mathrm{u}^{0}$ is directly proportional to the maximum dynamic response $\mathrm{D}^{0}$.

$$
\mathrm{u}^{0}=\Phi \Gamma \mathrm{D}^{0}
$$

Where $\mathrm{u}^{0}$ is the vector of maximum nodal displacements, $\mathrm{D}^{0}$ is the dynamic amplification vector and $\Gamma$ is the diagonal matrix of modal participation factors. To find appropriate expressions for $\Gamma$ and $\mathrm{D}^{0}$ we resort to the definition of the SMPF.

We begin by decomposing the vector of external forces into two components: a time invariant vector $f$ and a time dependent expression $\mathrm{p}(\mathrm{t})$.

$$
\mathrm{F}(\mathrm{t})=f \mathrm{p}(\mathrm{t})
$$

The vector $f$ can be expanded into a matrix of static forces $f^{s t}$ acting at node $\mathrm{j}$ in the mode $\mathrm{i}$; where the sum of the columns of $f^{s t}$ is equal to the magnitude of the $\mathrm{j}$-th component of the vector $f$ $[14,37]$.

$$
f_{j, i}^{s t}=\Gamma_{i, i} \phi_{j, i} \quad\left\{\begin{array}{l}
\text { for } i=1,2, \ldots, N \\
\text { for } j=1,2, \ldots, n
\end{array}\right.
$$

where:

$$
\Gamma=\operatorname{diag}\left(\Phi^{\mathrm{T}} \tilde{f}\right)
$$

When the eigenvectors are mass normalized, the vector of total static displacement can be found by the use of Hooke's law.

$$
\mathrm{u}_{\mathrm{j}}^{\mathrm{st}}=\sum_{\mathrm{i}=1}^{\mathrm{N}} \frac{\Gamma_{\mathrm{i}, \mathrm{i}}}{\omega_{\mathrm{i}}^{2}} \phi_{\mathrm{j}, \mathrm{i}} \quad \text { for } \mathrm{i}=1,2, \ldots, \mathrm{N}
$$

The vector of total static displacement can then be related to the vector of total dynamic displacements by introducing (9) into (2).

$$
u_{j}^{o}=u_{j}^{s t} \omega_{i}^{2} D_{i}^{o}
$$

Depending on the form of the excitation force $p(t)$, a particular method can be selected to find an analytical solution for $\mathrm{D}^{0}$, as explained below.

\section{A. Steady-state}

From [36], a dynamic amplification factor $\mathrm{R}_{\mathrm{i}}$ is defined as the ratio between the amplitude of the dynamic response $D_{i}^{o}$ and the amplitude of the static response $D_{i}^{\text {st }}$.

$$
\mathrm{R}_{\mathrm{i}}=\frac{\mathrm{D}_{\mathrm{i}}^{\mathrm{o}}}{\mathrm{D}_{\mathrm{i}}^{\mathrm{st}}}
$$

Where the amplitude of the static response can be found by neglecting the contribution of the acceleration and the velocity terms in the well-known mass-normalized modal equations of motion.

$$
\mathrm{D}_{\mathrm{i}}^{\mathrm{st}}=\frac{1}{\omega_{i}^{2}}
$$

And the dynamic amplification factor for an undamped single degree of freedom (SDOF) system is given by (13), when the unitary periodic function $\mathrm{p}(\mathrm{t})$ is equal to 1 .

$$
\mathrm{R}_{\mathrm{i}}=\frac{1}{\left(\omega_{\mathrm{i}}^{2}-\omega^{2}\right)}
$$

By introducing (11-13) into (10) we find an inexpensive expression that allows to estimate the maximum amplitude of the nodal physical displacements during steady-state vibration.

$$
\mathrm{u}^{\mathrm{o}}=\mathrm{u}^{\mathrm{st}} \mathrm{R}
$$

To compare the output of this method with respect to others, a steady-state modal participation fraction $\mathrm{L}_{\mathrm{i}}^{\text {st }}$ is defined in (15). This fractional number allows to compare the relative amplitudes of the modal responses $\mathrm{q}_{\mathrm{i}}$ for a given design and loading condition.

$$
\mathrm{L}_{\mathrm{i}}^{\mathrm{st}}=\frac{\left|\Gamma_{\mathrm{i}} \mathrm{D}_{\mathrm{i}}^{\mathrm{o}}\right|}{\sum_{\mathrm{i}}^{\mathrm{N}}\left|\Gamma_{\mathrm{i}} \mathrm{D}_{\mathrm{i}}^{\mathrm{o}}\right|}=\frac{\left|\Gamma_{\mathrm{i}} /\left(\omega_{\mathrm{i}}^{2}\left(\omega_{\mathrm{i}}^{2}-\omega^{2}\right)\right)\right|}{\sum_{\mathrm{i}}^{\mathrm{N}}|| \Gamma_{\mathrm{i}} /\left(\omega_{\mathrm{i}}^{2}\left(\omega_{\mathrm{i}}^{2}-\omega^{2}\right)\right) \mid}
$$

\section{B. Transient}

The steady participation factors neglect the contribution of the transient components of the time domain solution. When the global maximum is of particular interest, we aim to estimate the maximum dynamic amplitude response.

$$
\mathrm{D}_{\mathrm{i}}^{\max }=\max (\mathrm{D}(\mathrm{t}))
$$

The transient participation fraction $L_{i}^{t s}$ can then be defined similarly to the steady participation fraction as the ratio between the maximum amplitude of the harmonic solution and the sum of the maximum amplitude of all the modes retained.

$$
\mathrm{L}_{\mathrm{i}}^{\mathrm{ts}}=\frac{\left|\Gamma_{\mathrm{i}} \mathrm{D}_{\mathrm{i}}^{\max }\right|}{\sum_{\mathrm{i}}^{\mathrm{N}}\left|\Gamma_{\mathrm{i}} \mathrm{D}_{\mathrm{i}}^{\max }\right|}
$$

\section{Internal loads Participation Factor}

For point loads, the element internal force is computed as the product of the element stiffness matrix and the corresponding 
displacement vector of the nodes that conform the element in the global coordinate system.

When the element and global coordinate systems do not coincide a rotation matrix, $\mathrm{C}^{\mathrm{T}}$, is necessary to ensure that the internal forces computed, $\mathrm{N}_{\mathrm{e}}$, are expressed in the element coordinate system.

$$
\mathrm{N}_{\mathrm{e}}(\mathrm{t})=\mathrm{C}^{\mathrm{T}} \mathrm{K}_{\mathrm{e}} \mathrm{U}_{\mathrm{e}}(\mathrm{t})
$$

Where $\mathrm{K}_{\mathrm{e}}$ and $\mathrm{U}_{\mathrm{e}}(\mathrm{t})$ are the element stiffness matrix and the vector of nodal displacements in the global coordinate system, respectively.

We can expand (18) into its modal components as:

$$
\mathrm{N}_{\mathrm{e}}(\mathrm{t})=\mathrm{C}^{\mathrm{T}} \mathrm{K}_{\mathrm{e}} \Phi_{\mathrm{e}} \mathrm{q}(\mathrm{t})=\mathrm{C}^{\mathrm{T}} \mathrm{K}_{\mathrm{e}} \Phi_{\mathrm{e}} \Gamma \mathrm{D}(\mathrm{t})
$$

The magnitude of $\Gamma \mathrm{D}(\mathrm{t})$, determines the dominance of a mode in the solution, whereas the product $\Sigma_{\mathrm{e}}=\mathrm{C}^{\mathrm{T}} \mathrm{K}_{\mathrm{e}} \Phi_{\mathrm{e}}$ determines the impact of such mode in the $\mathrm{r}$ component of the element load where $r \in\{1: 6\}$. A participation factor can then be defined as:

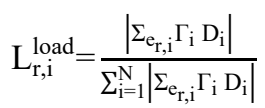

\section{CLASS II}

The following definitions intend to assess inexpensively the relative importance of a mode and to ensure that a sufficient number of eigenvalues are retained to recover the solution in the physical domain. The most widely used definition is that of the modal mass participation factor, in practice modes with negligible modal effective mass are removed from the equations of motion, since its participation to the overall response is small [20,38].

However other definitions have been proposed, for instance, Wilson [25] used a mode participation factor similar to (8) to estimate which modes should be retained in a subsequent mode superposition analysis. Carlbom et al. [26] presented four criteria to select important modes during the dynamic analysis of rail vehicles, and recently, Kammer, et.al. [39] proposed a new definition to select important elastic modes in free-free systems. In the following subsections we present most common definitions found in literature relevant to structural analysis.

\section{A. Modal Mass Participation Factor}

The modal effective mass was initially derived for a single degree of freedom system subject to an enforced motion through the base. The reaction force at the base $F_{\text {base }}$ due to the enforced acceleration $\ddot{U}_{0}$ in the frequency domain is given by:

$$
F_{\text {base }}(\omega)=m\left[1+\left(\frac{\omega}{\omega_{n}}\right)^{2} H\left(\frac{\omega}{\omega_{n}}\right)\right] \ddot{U}_{o}(\omega)
$$

In this context the effective mass of the system is $\mathrm{m}$ and it is directly proportional to the reaction force at the base. Thus, a large modal effective mass indicates a large inertial force.

The above analysis has been generalized to unconstrained multi-degree of freedom systems (MDOF) through a CraigBrampton transformation matrix $[9,38]$, and the resulting equation is (22), where $M$ is the mass matrix. This equation, relates the rigid-body (or unconstrained) modes $\Phi^{\mathrm{r}}$ with the elastic modes $\Phi^{\mathrm{e}}$.

$$
\Gamma^{\mathrm{mm} \mathrm{T}}=\Phi^{\mathrm{rT}} \mathrm{M} \Phi^{\mathrm{e}}
$$

We can prove that the product of the modal mass participation factors $\Gamma^{\mathrm{mm}}$ divided by the modal mass $M_{q}$ is identical to the mass matrix of unconstrained degrees of freedom. This, expressed in component form is the so called modal effective mass $\mathrm{M}^{\text {eff }}$.

$$
\mathrm{M}_{\mathrm{p}, \mathrm{i}}^{\mathrm{eff}}=\frac{\Gamma_{\mathrm{p}, \mathrm{i}}^{\mathrm{mm} \mathrm{T}} \Gamma_{\mathrm{i}, \mathrm{p}}^{\mathrm{mm}}}{\mathrm{M}_{\mathrm{i}, \mathrm{q}}}
$$

Where the subscript $p$ indicates the number of unconstrained elastic degrees of freedom (DoF) and $i$ is the number of modes retained in the analysis.

Though the definition of the modal effective mass has been derived using a free-free MDOF system, an important point should be kept in mind: the modal characteristics of the elastic degrees of freedom must be computed with respect to a boundary DoF, which should be constrained for the analysis $[6,8,38]$. Otherwise, the results extracted using the method described above are ill-founded and the participation of the elastic DoF's will tend to be zero.

Finally, for comparison purposes with other methods we can define an effective modal mass fraction $\mathrm{L}_{\mathrm{p}, \mathrm{i}}^{\mathrm{mm}}$ as the ratio between the effective modal mass and the total mass of the system:

$$
\mathrm{L}_{\mathrm{p}, \mathrm{i}}^{\mathrm{mm}}=\frac{\mathrm{M}_{\mathrm{p}, \mathrm{i}}^{\mathrm{eff}}}{\sum_{\mathrm{j}=1}^{\mathrm{n}} \mathrm{M}_{\mathrm{j}}}
$$

\section{B. Free-Free Participation Factor}

Recently [39], presented a new definition to estimate the relative importance of each mode for a free-free system, based on the concept of Effective Interface Mass (EIM), commonly used in the dynamic analysis of subsystems that will be attached to larger assemblies.

The equations of motion for a dynamic free-free system in matrix form are described as:

$$
\ddot{\mathrm{q}}(\mathrm{t})+\omega_{i}^{2} \mathrm{q}(\mathrm{t})=\Phi^{\mathrm{T}} f \mathrm{p}(\mathrm{t})
$$

Using equation (1) to recover the physical displacements from (25) and solving for the physical acceleration, we obtain:

$$
\ddot{\mathrm{u}}(\mathrm{t})=\Phi \ddot{\mathrm{q}}(\mathrm{t})=\Phi \Phi^{\mathrm{T}} f p(\mathrm{t})-\Phi \omega_{\mathrm{i}}^{2} \mathrm{q}(\mathrm{t})
$$

In (26), the modes that are strongly excited by the external force vector are, in consequence, expected to contribute strongly to the physical acceleration at the nodes. Thus, we can define a free-free modal participation factor $\Gamma_{i}^{f r e e}$ as the trace of the product between two eigenvectors.

$$
\Gamma_{\mathrm{i}}^{\mathrm{free}}=\operatorname{tr}\left(\Phi_{\mathrm{i}} \Phi_{\mathrm{i}}^{\mathrm{T}}\right)
$$

Since our interest lies in evaluating the relative importance of the elastic modes, the free-free participation factor $\mathrm{L}_{i}^{\text {free }}$ should be compared against the elastic degrees of freedom only.

$$
\Gamma^{\text {elas }}=\operatorname{tr}\left(\Phi^{\text {elas }} \Phi^{\text {elas }}{ }^{\mathrm{T}}\right)
$$


Thus, the free effective mass is defined as the fraction between the free-free modal participation factors and the trace of the product of the matrix of elastic eigenvectors.

$$
\mathrm{L}_{\mathrm{i}}^{\text {free }}=\frac{\Gamma_{\mathrm{i}}^{\text {free }}}{\Gamma^{\text {elas }}}
$$

\section{Modal Strain Energy Participation Factor}

Several authors [40-41] have proposed the use of the element modal strain energy $\mathrm{MSE}_{\mathrm{ei}}$ as assessment criteria. The element modal strain energy is evaluated at each element stiffness submatrix $\mathrm{K}_{\mathrm{e}}$ in the global coordinate system.

$$
\mathrm{MSE}_{\mathrm{ei}}=\frac{1}{2} \Phi_{\mathrm{e}, \mathrm{i}}^{\mathrm{T}} \mathrm{K}_{\mathrm{e}} \Phi_{\mathrm{e}, \mathrm{i}}
$$

Furthermore, the authors in [40] defined a modal strain participation fraction as the ratio between the strain energy and the kinetic energy of the system in free vibration.

$$
\mathrm{L}_{\mathrm{ei}}^{\mathrm{MSE}}=\frac{\Phi_{i}^{\mathrm{T}} \mathrm{K}_{\mathrm{e}} \Phi_{\mathrm{i}}}{\Phi_{\mathrm{i}}^{\mathrm{T}} \mathrm{M} \Phi_{\mathrm{i}} \omega_{\mathrm{i}}^{2}}
$$

A large modal strain fraction at the element e, at the i-th mode indicates that the element is a major load carrying component for such mode. Thus, any structural modifications in that element will produce significant changes in the modal characteristics of the structure and vice versa.

\section{CAse Study}

A simple 5-degree of freedom model was created to assess the performance of each of the methods described above. As shown in figure (1), the model consists of a five DOFs cantilever beam. Each beam element has a stiffness of $8700[\mathrm{~N} / \mathrm{m}]$ and a $5 \%$ modal critical damping is also considered.

The model is excited by a force $f$ of magnitude $100 \mathrm{~N}$ applied at the $5^{\text {th }}$ node with a periodic function and an excitation frequency of $5 \mathrm{rad} / \mathrm{s}$ as shown in (32). This analysis can be seen as a simplified aircraft wing under windmilling incident.

The modal equation of motion is solved in the time domain using Runge-Kutta's numerical method [52] and the result is presented in figure 2.

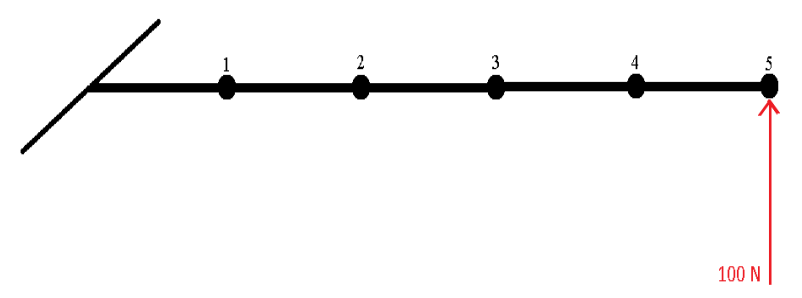

Figure 1. Case study: cantinlever beam

$$
p(t)=\sin (\omega t)
$$

The modal steady-state $\mathrm{L}_{\mathrm{i}}^{\text {st }}$, transition $\mathrm{L}_{i}^{t s}$, modal effective mass $\mathrm{L}_{i}^{m m}$ and free-free $\mathrm{L}_{i}^{f f}$ participation fractions are compute using equations (15), (17), (24) and (29), respectively, and the results are shown in figure 3 . This figure gives an idea of the difference in magnitude obtained with each method, however the results should be interpreted independently.
Both, the steady-state $\mathrm{L}_{\mathrm{i}}^{\text {st }}$ and the transition $\mathrm{L}_{i}^{t s}$ participation fractions are estimates of the amplitude of the modal response, therefore the percentage of participation is well in agreement with figure 2: the amplitude of the first mode is very large in comparison to that of the fifth mode for the given excitation frequency.

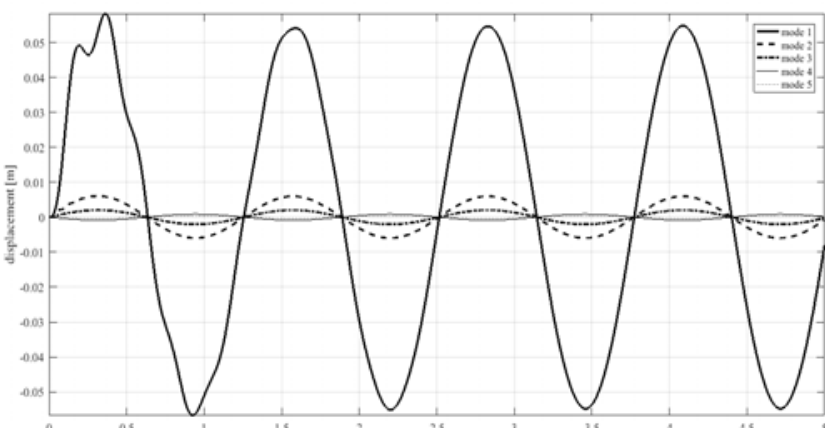

Figure 2. Modal response.

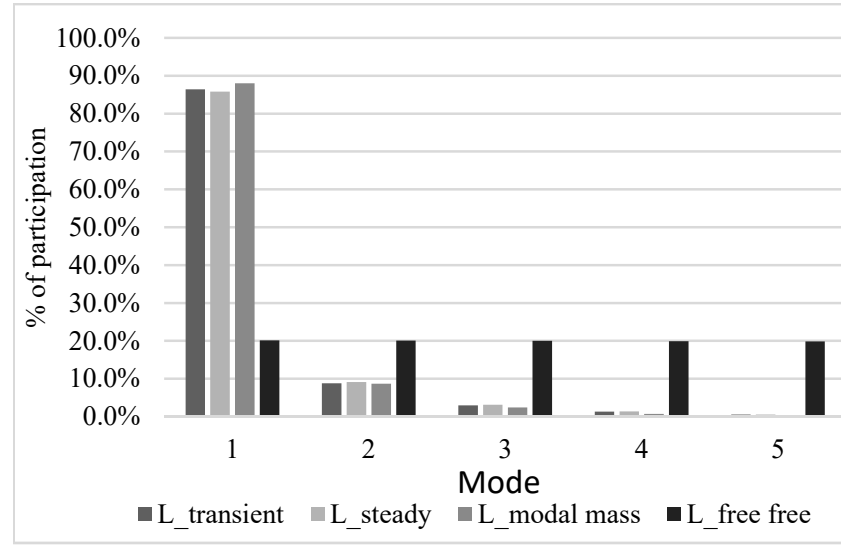

Figure 3. Modal participation factors.

The modal effective mass participation factor is also in agreement with figure 2, a large modal effective mass indicates that a particular mode has a large modal inertial component, and thus it is easier to excite.

The estimated free-free participation fractions are mostly in disagreement with the rest of the participation fractions computed, since its magnitude reveals that the participation of all modes is almost identical. Note that the definition of $L_{i}^{f f}$ is simply the trace of the modal residues, i.e. how the node i-th responds to an excitation applied at the $\mathrm{j}$-th node.

Using both, the steady-state and transition modal response participation factors, the contribution of each mode to the internal load was estimated using equation (20). The modal strain energy was also computed using equation (31). In order to compare the three sets of results, the values of each matrix were normalized with respect to the component with the largest magnitude and the results are displayed in figure 4.

Take for example figure 6 (d), according to the modal strain energy, it appears that the second mode becomes more relevant to the response of element 4 , thus, any structural modification 
that could impact the behavior of the second mode would also have a major impact in the magnitude of the load experienced by element 4 . However, the results obtained using equation (32) are in disagreement with those of the modal strain energy, which indicates that the internal load is dominated by the first elastic mode.
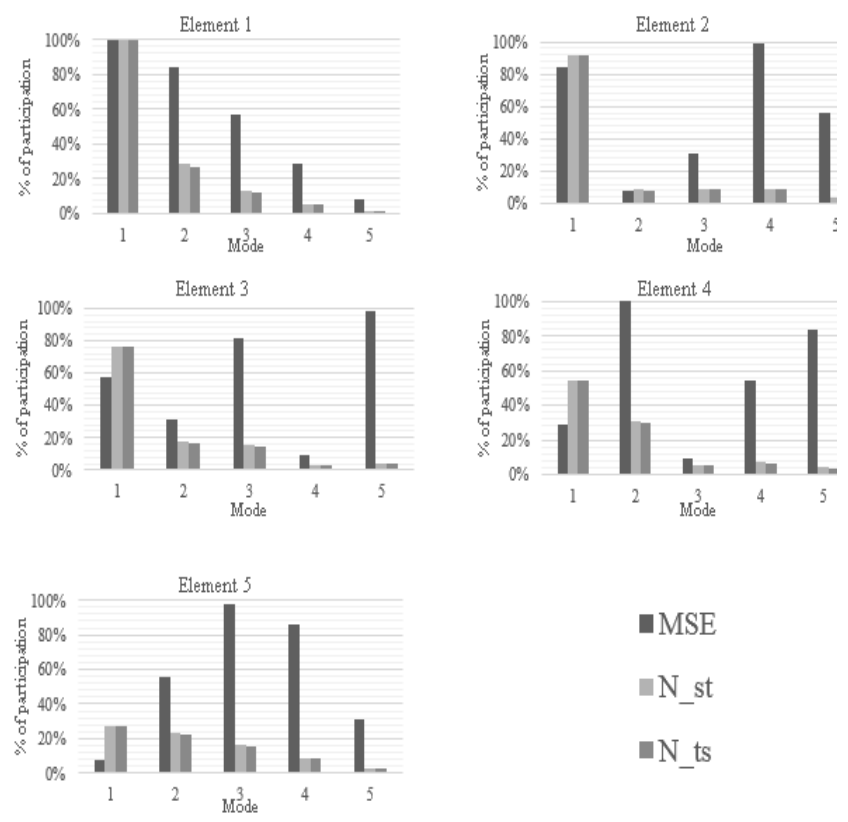

Figure 4. Internal load modal participation factors.

Using the steady and the transient modal response participation factors, the peak in plane shear force was recovered using equation (19) with (15) and (17), respectively. Table I shows a comparison and percentage error between the recovered load and the actual peak load computed using MSC NASTRAN [53].

TABLE I. PEAK SHEAR FORCE PER ELEMENT

\begin{tabular}{|c|c|c|c|c|c|}
\hline \multicolumn{7}{|c|}{ Peak shear force per element } \\
\hline \multirow{2}{*}{ Element } & $\begin{array}{c}\text { Actual } \\
\text { Peak } \\
\text { Load }\end{array}$ & $\begin{array}{c}\text { Load } \\
\Gamma_{i} D_{i}{ }^{s t}\end{array}$ & \%error & $\begin{array}{c}\text { Load } \\
\Gamma_{i} D_{i}{ }^{t s}\end{array}$ & \%error \\
\hline 1 & 55.8422 & 51.9211 & $7.02 \%$ & 60.3657 & $8.10 \%$ \\
\hline 2 & 55.2437 & 51.7904 & $6.25 \%$ & 47.0600 & $-14.81 \%$ \\
\hline 3 & 54.1775 & 51.5293 & $4.89 \%$ & 52.3115 & $-3.44 \%$ \\
\hline 4 & 52.6880 & 51.1385 & $2.94 \%$ & 67.5420 & $28.19 \%$ \\
\hline 5 & 50.8545 & 50.6189 & $0.46 \%$ & 37.18662 & $-26.88 \%$ \\
\hline
\end{tabular}

The percentage error of the steady-state modal response participation factors $\mathrm{L}_{\mathrm{i}}^{\text {st }}$ is due to the omission of the transient terms. Since the peak load occurs during the transition period, the load recovered corresponds in fact to the actual steady-state in-plane shear force.

On the other hand, error of the peak load recovered using the transient modal response participation factor $\mathrm{L}_{i}^{t s}$ was found to be significantly larger. This is due to an implicit assumption in (16): the peak of all the modal responses are in phase, thus the method tends to either over or underestimate the value of the peak load.

\section{CONCLUSIONS}

The first class of modal response participation factors have the potential to be used as reanalysis tools. When the excitation force is periodic and the interest lies in solving for the steadystate response, the $\mathrm{L}_{\mathrm{i}}^{\text {st }}$ fraction provides accurate results.

The second class of modal response participation factors are independent of the excitation and depend solely on the mass and stiffness distribution, in this sense, this type of factors should not be used as a measure of the relative amplitude of the modal responses. Instead, their application should be restricted, for example, in the case of the modal effective mass fraction, to determine the number of modes to be retained in the analysis (except when the model has an enforced acceleration through the base $[2,3])$. Such methodology can serve as an inexpensive response tracking mechanism in complex structures during design optimization.

The modal strain energy, on the other hand, is an inexpensive expression that relates the mass and stiffness distribution of the structure to its modal characteristics. This expression can be useful to visualize and correlate how modifying the physical properties at element level can impact the behavior of a specific mode.

\section{REFERENCES}

[1] Lau, G. K., \& Du, H. (2005). Topology optimization of head suspension assemblies using modal participation factor for mode tracking. Microsystem Technologies, 11(12), 1243-1251.

[2] Miranda, E. (1999). Approximate seismic lateral deformation demands in multistory buildings. Journal of Structural Engineering, 125(4), 417-425.

[3] Oh, B. K., Kim, M. S., Kim, Y., Cho, T., \& Park, H. S. (2015). Model updating technique based on modal participation factors for beam structures. Computer-Aided Civil and Infrastructure Engineering, 30(9), 733-747.

[4] Kobori, T., Koshika, N., Yamada, K., \& Ikeda, Y. (1991). Seismic-responsecontrolled structure with active mass driver system. Part 1: Design. Earthquake Engineering \& Structural Dynamics, 20(2), 133-149.

[5] Park, H. G., Eom, T., \& Lee, H. (2007). Factored modal combination for evaluation of earthquake load profiles. Journal of Structural Engineering, 133(7), 956-968.

[6] Chopra, A. K., \& Chintanapakdee, C. (2001). Drift spectrum vs. modal analysis of structural response to near-fault ground motions. Earthquake Spectra, 17(2), 221-234.

[7] Palermo, M., Silvestri, S., Gasparini, G., \& Trombetti, T. (2015). Seismic modal contribution factors. Bulletin of Earthquake Engineering, 13(10), 2867-2891.

[8] Ghahari, S. F., Abazarsa, F., Ghannad, M. A., \& Taciroglu, E. (2013). Response-only modal identification of structures using strong motion data. Earthquake Engineering \& Structural Dynamics, 42(8), 1221-1242.

[9] Girard, A., \& Roy, N. A. (1997). Modal effective parameters in structural dynamics. Revue Européenne des éléments finis, 6(2), 233-254.

[10] Igusa, T., Kiureghian, A. D., \& Sackman, J. L. (1984). Modal decomposition method for stationary response of non-classically damped systems. Earthquake engineering \& structural dynamics, 12(1), 121-136.

[11] Whittaker, A. S., Constantinou, M. C., Ramirez, O. M., Johnson, M. W., \& Chrysostomou, C. Z. (2003). Equivalent lateral force and modal analysis procedures of the 2000 NEHRP Provisions for buildings with damping systems. Earthquake Spectra, 19(4), 959-980.

[12] Miranda, E., \& Taghavi, S. (2005). Approximate floor acceleration demands in multistory buildings. I: Formulation. Journal of structural engineering, 131(2), 203-211.

[13] Wilson, E. L., Der Kiureghian, A., \& Bayo, E. P. (1981). A replacement for the SRSS method in seismic analysis. Earthquake Engineering \& Structural Dynamics, 9(2), 187-192. 
[14] Romera, L. E., \& Hernandez, S. (1999). An improved technique for modal contribution factors of dynamic responses. In AIAA/ASME/ASCE/AHS/ASC Structures, Structural Dynamics, and Materials Conference and Exhibit, 40 th, St. Louis, MO (pp. 439-445).

[15] Arief, A., Nappu, M. B., Nizar, A., \& Dong, Z. Y. (2009). Determination of DG allocation with modal participation factor to enhance voltage stability.

[16] Gebreselassie, A., \& Chow, J. H. (1994). Investigation of the effects of load models and generator voltage regulators on voltage stability. International Journal of Electrical Power \& Energy Systems, 16(2), 83-89.

[17] Arief, A., Nappu, M. B., Dong, Z. Y., \& Arief, M. (2010, October). Under voltage load shedding incorporating bus participation factor. In IPEC, 2010 Conference Proceedings(pp. 561-566). IEEE.

[18] Hashlamoun, W. A., Hassouneh, M. A., \& Abed, E. H. (2009). New results on modal participation factors: Revealing a previously unknown dichotomy. IEEE Transactions on Automatic Control, 54(7), 1439-1449.

[19] MacNeal, R. (1972). The NASTRAN Theoretical Manual (level 15.5): Msr-40. MacNeal-Schwendler Corporation.

[20] Irvine, T. (2013). Effective modal mass and modal participation factors. Available on the web on site: http://www. vibrationdata. com/tutorials2/ModalMass. pdf.(last access on march 7 2007).

[21] Girard, A., \& Roy, N. A. (1997). Modal effective parameters in structural dynamics. Revue Européenne des éléments finis, 6(2), 233-254.

[22] Kuhar, E. J., \& Stahle, C. V. (1974). Dynamic transformation method for modal synthesis. AIAA Journal, 12(5), 672-678.

[23] Chen, J. T., Chen, K. H., Chen, I. L., \& Liu, L. W. (2003). A new concept of modal participation factor for numerical instability in the dual BEM for exterior acoustics. Mechanics Research Communications, 30(2), 161174.

[24] Chen, J. T., Hong, H. K., \& Yeh, C. S. (1995). Modal reaction method for modal participation factors in support motion problems. International Journal for Numerical Methods in Biomedical Engineering, 11(6), 479490 .

[25] Wison, E. L., Yuan, M. W., \& Dickens, J. M. (1982). Dynamic analysis by direct superposition of Ritz vector. Earthquake engineering and structural dynamics, 10, 813-821.

[26] Carlbom, P. F. (2001). Combining MBS with FEM for rail vehicle dynamics analysis. Multibody System Dynamics, 6(3), 291-300.

[27] Salmonte, A. J. (1982). Considerations on the residual contribution in modal analysis. Earthquake Engineering \& Structural Dynamics, 10(2), 295-304.

[28] Photiadis, D. M., Houston, B. H., Liu, X., Bucaro, J. A., \& Marcus, M. H. (2002). Thermoelastic loss observed in a high Q mechanical oscillator. Physica B: Condensed Matter, 316, 408-410.

[29] Wallrapp, O., \& Wiedemann, S. (2002). Simulation of deployment of a flexible solar array. Multibody System Dynamics, 7(1), 101-125.

[30] Yun, C. B., \& Bahng, E. Y. (2000). Substructural identification using neural networks. Computers \& Structures, 77(1), 41-52.

[31] Van Lagenhove, T., \& Brughmans, M. (1999, June). Using MSC/NASTRAN and LMS/PRETEST to find an optimal sensor placement for modal identification and correlation of aerospace structures. In 2nd MSC Aerospace Conference (pp. 7-11).

[32] Asmussen, J. C. (1997). Modal analysis based on the random decrement technique. Department of Building Technology and Structural Engineering University of Aalborg.

[33] Fang, S. M., \& NIEDZWECKI, J. M. (2014). Comparison of airfoil and ribbon fairings for suppression of flow-induced vibrations. International Journal of Computational Methods and Experimental Measurements, 2(1), 30-45.
[34] Przekop, A., Rizzi, S. A., \& Groen, D. S. (2006). Nonlinear acoustic response of an aircraft fuselage sidewall structure by a reduced-order analysis.

[35] Zhang, L., Brincker, R., \& Andersen, P. (2001, February). Modal indicators for operational modal identification. In 19th International Modal Analysis Conference (IMAC), Kissimmee, Florida.

[36] Chopra, A. K. (2001). Dynamics of structures: Theory and applications. Prentice Hall.

[37] Chopra, A. K. (1996). Modal analysis of linear dynamic systems: physical interpretation. Journal of structural engineering, 122(5), 517-527.

[38] Wijker, J. J. (2008). Spacecraft structures. Springer Science \& Business Media.

[39] Kammer, D. C., Cessna, J., \& Kostuch, A. (2005). An Effective Mass measure for selecting free-free target modes. In Proceedings of the $23 \mathrm{rd}$ International Modal Analysis Conference, Orlando, Florida.

[40] Li, H., Yang, H., \& Hu, S. L. J. (2006). Modal strain energy decomposition method for damage localization in 3D frame structures. Journal of engineering mechanics, 132(9), 941-951.

[41] Li, L., Hu, Y., \& Wang, X. (2013). Numerical methods for evaluating the sensitivity of element modal strain energy. Finite Elements in Analysis and Design, 64, 13-23.

[42] Zou, X. K., \& Chan, C. M. (2005). An optimal resizing technique for seismic drift design of concrete buildings subjected to response spectrum and time history loadings. Computers \& structures, 83(19-20), 16891704.

[43] Chen, H. P. (2006). Efficient methods for determining modal parameters of dynamic structures with large modifications. Journal of Sound and Vibration, 298(1-2), 462-470.

[44] Lou, M., \& Chen, G. (2003). Modal perturbation method and its applications in structural systems. Journal of engineering mechanics, 129(8), 935-943.

[45] To, W. M., \& Ewins, D. J. (1991). Non-linear sensitivity analysis of mechanical structures using modal data. Proceedings of the Institution of Mechanical Engineers, Part C: Mechanical Engineering Science, 205(1), 67-75.

[46] Massa, F., Lallemand, B., \& Tison, T. (2015). Multi-level homotopy perturbation and projection techniques for the reanalysis of quadratic eigenvalue problems: The application of stability analysis. Mechanical Systems and Signal Processing, 52, 88-104.

[47] Ki-Ook, K., \& Wallerstein, D. V. (1988). Modal design sensitivities for multiple eigenvalues. Computers \& structures, 29(5), 755-762.

[48] Saito, A., Suzuki, H., Kuroishi, M., \& Nakai, H. (2015). Efficient forced vibration reanalysis method for rotating electric machines. Journal of Sound and Vibration, 334, 388-403.

[49] Dutta, A., \& Ramakrishnan, C. V. (1997). Error estimation in finite element transient dynamic analysis using modal superposition. Engineering Computations, 14(1), 135-158.

[50] Grissom, M. D., Belegundu, A. D., \& Koopmann, G. H. (2005). A reduced eigenvalue method for broadband analysis of a structure with vibration absorbers possessing rotatory inertia. Journal of sound and vibration, 281(3-5), 869-886.

[51] Constans, E. W., Belegundu, A. D., \& Koopmann, G. H. (2000). Optimally designed shell enclosures with tuned absorbers for minimizing sound power. Optimization and Engineering, 1(1), 67-86.

[52] Butcher, J. C. (1987). The numerical analysis of ordinary differential equations: Runge-Kutta and general linear methods.

[53] Nastran, M. S. C. (2004). Quick reference guide. MSC. SOFTWARE, 1. 